\title{
Quels sont les Éléments Essentiels de la Recherche Valable? - Les Problèmes de «Données » et leur Cueillette dans les Contextes Interculturels
}

Judith K. Bernhard

Ryerson University

digital.library.ryerson.ca/object/23

Please Cite:

Bernhard, J. K. (2010). Quels sont les éléments essentiels de la recherche valable? Les problèmes de " données » et leur cueillette dans les contextes interculturels. Perspectives de Soutien à la Famille, 3(Printemps), 27-32.

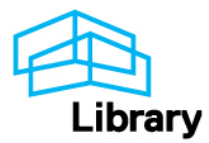




\title{
Quels sont les éléments essentiels de la recherche valable?
}

\section{Le problème des « données » et leur cueillette dans les contextes interculturels}

\author{
Judith K. Bernhard, Ph.D.
}

\begin{abstract}
$\nabla$ Résumé
La professeure Judith Bernhard enseigne à la School of Early Childhood Education de l'Université Ryerson. Elle s'appuie sur sa propre expérience de la recherche sur les familles transnationales pour illustrer les difficultés de la collecte de données et pour alimenter ainsi la réflexion sur les pratiques dans ce domaine. Elle commence par exposer sa propre histoire d'immigrante et d'universitaire. Elle vit elle-même les tensions entre la défense des intérêts des familles et son rôle comme chercheuse universitaire. Elle utilise deux études de cas pour illustrer certaines de ces difficultés. La première est celle de points de vue divergents sur le rôle du parent dans le cas d'une jeune adolescente dont la famille était originaire du Salvador. L'enseignante de la jeune fille et sa famille ne partageaient pas les mêmes opinions sur ses droits et responsabilités à cause de leurs valeurs culturelles. La professeure Bernhard explique que ces divergences d'opinions étaient également représentées au sein de l'équipe de chercheurs. Ses propres racines latino-américaines lui ont permis de comprendre la position de la famille et sa formation universitaire de comprendre les buts poursuivis par l'enseignante. L'équipe a rédigé un rapport qui ne reflétait pas totalement la tension entre ces cultures. La professeure Bernhard a cependant conclu que les chercheurs doivent prêter plus attention à leur propre formation professionnelle et acculturation qui sont d'importants marqueurs dans le processus de recherche. La deuxième étude de cas qu'elle présente expose les difficultés qu'elle a rencontrées en utilisant des entrevues pour enquêter sur le vécu de mères somaliennes réfugiées. Le premier enquêteur, bien que ce soit un chercheur chevronné avec des racines immigrantes, s'est avéré inacceptable pour les familles somaliennes parce qu'il était de sexe masculin. La deuxième personne choisie est originaire de la communauté somalienne et a été formée par les chercheurs pour obtenir le consentement éclairé et pour conduire des entrevues normalisées. La professeure explique comment les perceptions et les normes culturelles interdisent d'appliquer ces approches normalisées avec la population de l'enquête, ce qui empêche la publication d'un rapport de recherche crédible. Du fait même, les informations sur la situation de ces familles et sur leur point de vue ne parviendront pas aux professionnels et aux décideurs dont les décisions influencent leur vie. La professeure Bernhard incite le milieu de la recherche à réfléchir aux modalités de définition des « données » significatives et des meilleures méthodes à employer pour les collecter.
\end{abstract}




\section{Mon « moi » subjectif}

Les différences culturelles et leur gestion ont fait partie de ma vie par nécessité, mais ont été également source de gratification et d'enrichissement. J'ai acquis mon expérience de la traversée des frontières d'abord avec ma famille, ensuite quand j'étais étudiante, puis enseignante, et, enfin, mère. Je suis maintenant professeure et directrice d'un programme de maîtrise en études de la petite enfance. Je suis également migrante. Née au Chili, j'ai ensuite fait mon " pays » du Puerto Rico, de la République dominicaine et du Canada.

Grâce à mes déplacements d'un continent à un autre, à l'apprentissage de nouvelles langues, de nouvelles visions du monde et de nouveaux ensembles de "valeurs fondamentales ", j'ai compris la nature arbitraire des règles et des règlements qui servent à classer, trier et gérer les personnes.

J'ai été la chercheure principale de plusieurs projets destinés à documenter les difficultés rencontrées par les familles migrantes quand leurs enfants essaient de trouver le juste équilibre entre la réussite dans les écoles nord-américaines et la conservation de leur identité culturelle d'origine et de la langue de leur famille. Mes collègues dans ces projets et moi-même avons toujours cherché à saisir l'expérience vécue par les « sujets » de nos travaux de recherche afin de faire évoluer les pratiques éducatives et politiques grâce à ces nouvelles connaissances. Nous avons, par conséquent, souhaité que nos constatations viennent en aide aux éducateurs dans les grandes difficultés auxquelles nous les savons confrontés dans leur travail auprès d'élèves qu'ils perçoivent comme différents et, souvent, comme problématiques.

Nous avons tout fait, dans notre travail, pour éviter d'opposer les faits objectifs et subjectifs. Nous avons cherché à diagnostiquer de façon systémique les problèmes caractéristiques du vécu des personnes que nous avons étudiées. Nous avons procédé ainsi parce que nous croyons que la connaissance sociale existe toujours en relation avec une situation et un contexte.

C'est ainsi que nous avons toujours reconnu que la diversité de l'équipe de recherche est indispensable pour mettre en lumière le vécu de nos participants. Les membres de l'équipe de divers milieux culturels permettent au reste de l'équipe de comprendre le sens implicite des mots et des actes des participants. Notre spécialité en est encore au stade où la sélection d'une équipe multiethnique doit être délibérée et parfois même imposée, parce que nos établissements ne sont pas représentatifs de tous les groupes avec lesquels nous travaillons. Ma contribution au présent ouvrage sera axée sur certaines des difficultés auxquelles mes collègues et moi-même avons été confrontés en travaillant dans des équipes multiethniques et multidisciplinaires.

Quel est le sens de la «différence » pour moi? Au cours de ma vie, j'ai fait l'expérience des changements constants d'appartenance et d'allégeance. Comme le dit Mary Catherine Bateson,

...l'appartenance est artificielle, doit être construite... l'appartenance officialise et comble à la fois la séparation; elle est construite pour combler un fossé d'incompréhension, en dépendant toujours de la volonté de se joindre au mouvement commun et d'être modifié par lui. (1994, p. 62)

Depuis mon arrivée en Amérique du Nord, j'ai été classée dans de nombreuses catégories : celles des immigrants, des touristes, des migrants involontaires et, selon ma position par rapport à la frontière des Amériques, dans celles des Blanches, des Latino-américaines ou des Hispaniques. Quand je me trouve à un endroit, les valeurs et les manières de l'autre endroit me manquent et, pourtant, quand je me rends à cet autre endroit, je le trouve différent de mes souvenirs.

Quand j'ai immigré au Canada, au début je voulais adopter tout ce qui me paraissait moderne et progressif. Et pourtant, à cause de cette attitude, je me sentais souvent devenue étrangère à moi-même. Eva Hoffman décrit un sentiment comparable dans Lost in Translation. Elle décrit son départ de Cracovie pour le Canada à l'âge de 13 ans et la perte de contact avec son identité intime, ressentie peu de temps après.

Les pires sentiments de perte surgissent la nuit. Quand je suis allongée dans un lit étrange d'une étrange maison... j'attends le flot spontané du langage intérieur qui était mon dialogue intime la nuit et rien ne vient. Le polonais, en peu de temps, s'est atrophié et flétri, tout simplement parce qu'il est inutile. Ses mots ne s'appliquent pas à ce que je vis maintenant; ils ne sont pas contemporains des objets ou des visages ou de l'air même que je respire le jour. En anglais, les mots n'ont pas atteint ces couches de mon psychisme où un dialogue intime peut naître... Maintenant ces images et ce spectacle de mots sont partis, ce fil s'est brisé. Je n'ai pas de langage intérieur. (Hoffman, 1990, p. 107-108)

La difficulté de conserver ma subjectivité familière, tout en étant tiraillée et en désirant m'assimiler au nouvel ordre dominant, est difficile et rejaillit encore aujourd'hui sur chaque aspect de ma vie. Dans mon travail de chercheuse, je ressens les difficultés de ce tiraillement. Je suis honorée de faire entendre ma voix dans le milieu universitaire, mais il est parfois difficile de changer d'optique quand je tente de prendre à la fois la défense des familles et de mener une enquête universitaire.

La conception quantitative et dictée par les données des sciences sociales a souvent été critiquée. Diverses alternatives ont été proposées, dont des conceptions ethnographiques, qui, dit-on, saisissent une réalité qui n'est pas révélée par les «données objectives ». En revanche, comme les exemples ci-dessous l'illustrent, les méthodes de recherche valides et de collecte de données pouvant être considérées comme " objectives » (c.-à-d. pas simplement le fruit de l'imagination du chercheur) sont problématiques. Les problèmes rencontrés pour obtenir des participants des réponses informatives et sincères, ainsi que ceux

\section{8 perspectives}


rencontrés pour choisir les réponses à utiliser dans les rapports de recherche en respectant les principes éthiques ne se résolvent pas simplement par la conduite d'entrevues ethnographiques.

Bien que les problèmes des données des entrevues ne soient pas inconnus des anthropologues, les types de problèmes de recherche que nous exposons dans les études de la petite enfance n'ont pas retenu beaucoup l'attention. On n'a pas reconnu dans toute son ampleur le problème des mains liées et du clavier immobilisé des chercheurs en études de la petite enfance qui voudraient enquêter et publier des rapports sur des familles de la diversité, tout en s'assurant que ces enquêtes et ces rapports respectent les normes professionnelles des sciences sociales.

J'aimerais vous parler de ces questions, en les illustrant par deux études de cas. La première a trait à un projet de recherche avec des mères latino-américaines, la deuxième à un projet de recherche avec des mères somaliennes.

\section{Illustration un : Qui pouvons-nous représenter? L'histoire d'Isabel}

Le cadre de cette étude de cas est celui de mon entrevue avec une enseignante du centre-ville de Toronto. Nous parlons de son élève, Isabel Torres, 13 ans, née au Salvador et arrivée au Canada à l'âge de 10 ans. Je fais partie d'une équipe qui observe la vie de cette école en y participant depuis deux mois, en prenant des notes de recherche et en analysant les dossiers de l'école.

J'ai également pu me rendre à trois reprises au domicile de la famille Torres. Les quatre enfants Torres vivent avec leur mère, leur père et leur grand-mère paternelle dans un logement subventionné. Le père s'exprime dans un anglais très rudimentaire et la mère ne s'exprime qu'en espagnol. Le père travaille en postes de nuit et la mère et la grand-mère paternelle restent au domicile avec les enfants. La famille ne parle qu'espagnol à la maison et Isabel maîtrise l'espagnol avec beaucoup d'aisance.

En parlant avec moi des valeurs et des pratiques familiales, les parents d'Isabel ont affirmé que les liens étroits des membres de la famille sont essentiels. Ils sont persuadés qu'il est indispensable que les parents connaissent parfaitement les activités de leurs enfants, sachent où ils se trouvent à tout moment et connaissent les difficultés auxquelles les enfants font face dans leur vie.

...selon moi, un des parents doit rester à la maison avec les enfants, pour veiller à ce qu'ils mangent bien et surveiller ce qu'ils font. Mes enfants rentrent à la maison tous les jours pour le dîner... tous les jours ils prennent de la soupe... Maintenant, mon grand garçon est à l'école secondaire, donc il ne peut pas rentrer à la maison, mais j'ai un ami qui a un restaurant et il va y manger tous les jours.

Dans une entrevue séparée, l'enseignante d'Isabel me parle. Elle présente Isabel comme une élève solide, motivée et excellente dans toutes les disciplines. La contradiction entre les valeurs de l'enseignante et celles des parents d'Isabel apparaît cependant clairement dans ses propos.

... je pense qu'Isabel a très peur des conséquences chez elle... c'est peut-être pour cette raison qu'elle se comporte bien... je suis donc très prudente à l'égard de ce que je leur dis. Le père est toujours à l'école, il vient les chercher pour dîner et je trouve leur contrôle excessif. Son attitude est celle de quelqu'un qui régit tout, vous savez. Même la mère semble terrorisée. Il la cloître pieds nus et enceinte dans la cuisine, comme on dit, donc comment peut-elle aider les enfants? Pas plus tard que l'autre jour, j'ai appris [par le père] qu'Isabel avait écrit dans son journal intime qu'un garçon lui avait demandé de l'embrasser. Son journal intime est confidentiel... Il ne devrait pas le lire... il veut connaître le nom du garçon, maintenant.

\section{Interprétation de l'illustration un : aux prises avec les différences au sein de la communauté}

Les données précédentes ont alimenté une discussion interminable au sein de cette équipe de recherche. Cette équipe se composait de trois femmes et de deux hommes. Les femmes étaient moi-même, une femme du Ghana et une femme canadienne d'origine européenne. Un des hommes était Canadien, d'origine européenne, et l'autre, originaire du Ghana. D'après l'accord auquel notre équipe était parvenue, les données seraient analysées pendant nos séances de discussion. Cette méthode était nouvelle pour moi, parce qu'auparavant, quand j'avais collaboré avec d'autres personnes, j'avais toujours vécu le partage des tâches et le travail en solitaire sur ma petite partie du travail. La famille Torres et la réalité de sa situation, comme on disait, ont été analysées par les membres de l'équipe de recherche qui ont adopté la position de sujets. Que doit-on faire figurer dans le rapport de recherche?

Selon certains membres de l'équipe, Isabel est une enfant à risque. C'est une enfant effrayée par les techniques de terreur de son père autoritaire, disent-ils. Lingérence systématique de la famille dans les affaires des uns et des autres est jugée envahissante et excessivement sévère. Selon ces chercheurs, cette adolescente possède des droits individuels et son développement personnel est primordial. C'est une personne accomplie, capable, selon eux, de prendre ses propres décisions. Dans ces conditions, pour ces membres de l'équipe, les tentatives d'ingérence des adultes, même de ses propres parents, sont déplacées et oppressantes.

Selon d'autres membres de l'équipe, le rôle du père est normal, vu dans son contexte culturel. Isabel est, en contrepartie, le parfait exemple des avantages d'une approche biculturelle. Ils trouvent le père « récalcitrant ». Il est cependant important de comprendre le jugement qui explique l'emploi du terme « récalcitrant ». Il laisse entendre que ce que souhaite le père est en contradiction avec ce que souhaite l'enseignante et que celle-ci applique une grille 
universelle à Isabel. Voici, par exemple, ce que l'enseignante semble dire : «Voici une adolescente, ce sont ses tâches à réaliser pour son développement (une adulte en devenir qui a besoin de liberté). Ce dont elle a besoin, c'est d'un meilleur modèle familial, démocratique et centré sur l'enfant. »

D'un autre point de vue, il est cependant possible de concevoir que l'attitude du père n'est pas simplement une attitude d'opposition à l'enseignante. C'est plutôt la source de la force familiale. Étant donné la structure d'autorité de la famille, les « droits » d'une mineure à charge prennent peu d'importance dans ce modèle. C'est plutôt le bien-être de la famille, y compris celui de ses membres mineurs, qui est important. Un enfant de 13 ans n'est pas en âge de prendre des décisions sur des questions telles que les fréquentations. Le rôle du parent consiste à enseigner, guider et protéger l'enfant.

Le rôle de l'enseignante me préoccupait également. En communiquant ses opinions à Isabel, elle affirmait sa propre autorité et, par là même, sapait celle du père d'Isabel. Cette famille risquait d'être perturbée au plan émotif. Si l'enseignante réussissait à armer Isabel d'idées bien arrêtées sur ses droits, cela la conduirait inévitablement à s'affronter à son père et au reste de la famille. En d'autres termes, une question apparemment aussi mineure qu'empêcher le père de lire un journal intime pourrait sérieusement endommager cette structure familiale.

Dans nos discussions en équipe sur ce cas, mes collègues et moi-même avons rapidement compris que nos deux points de vue différents ne pourraient pas être totalement réconciliés ou harmonisés. Tous les regards se sont tournés vers moi, la membre latino-américaine de l'équipe. Je me suis cependant aperçue que mes réactions étaient (et sont toujours) partagées sur cette question. Une de mes réactions est inspirée par ma vision du monde de latino-américaine, partageant le but de la mère, à savoir essayer de s'adapter à la vie au Canada et de conserver sa culture et ses valeurs, ainsi que la cohésion de sa famille. Je reconnais que l'enseignante était influencée par les stéréotypes de la famille latino-américaine, et plus particulièrement, par les valeurs catholiques dans les domaines de la famille, de la régulation des naissances et de l'avortement. Je me pose la question suivante : si le père est présent tous les jours à l'école, pourquoi cette enseignante ne peut-elle pas utiliser les points forts de cette famille pour améliorer ses relations avec elle? Pourquoi toute cette méfiance des deux côtés?

Je comprends également la raison d'être de l'attitude de l'enseignante, une femme de la classe moyenne, ayant effectué de nombreuses années d'études, une féministe qui a choisi cette carrière et qui valorise le respect de la vie privée et les droits des enfants. Mon expérience de la formation pendant tant d'années dans des établissements d'enseignement postsecondaire euro-américains m'a fait abandonner ma vision du monde relationnelle. Je suis maintenant une professionnelle, qui applique les lois qui régissent les rapports de recherche. Bien que cette famille me soit reconnaissante de m'exprimer dans sa langue, il existe une distance entre nous qui ne peut pas être ignorée.

Lors de nos discussions en équipe, nous nous sommes rappelés aux uns et aux autres qu'il est impossible de juger le père d'Isabel indépendamment du contexte. Nous avons compris que, bien que nous définissions souvent les différences en fonction des genres et des classes sociales, nous devons prêter plus attention à notre propre formation professionnelle et à notre propre acculturation, qui sont d'importants marqueurs dont il faut tenir compte dans le processus de recherche. Il me semble que la structure de la famille doit être comprise et respectée. Je penchais du côté de la famille dans ce conflit, tout en étant consciente des sujets de préoccupation de l'enseignante. Ayant moi-même été enseignante, je connais bien ce "désir de ce qu'il y a de mieux pour l'enfant » et la défense des intérêts de l'enfant en lui donnant voix au chapitre. Je ne pense cependant pas que le rôle normal d'un enseignant consiste à se mettre à la place d'un enfant dans une famille ou de promouvoir la position de l'enfant en entraînant inévitablement des conséquences négatives pour l'enfant. L'aptitude de comprendre des visions du monde et des points de vue contradictoires m'a distanciée d'Isabel et de sa famille et j'ai dû me débattre dans les difficultés de la responsabilité d'être la latino-américaine de l'équipe.

Le renversement des rôles susceptible de se produire pour les enfants dans le cas d'Isabel peut rendre leur apprentissage difficile. Tous ceux qui travaillent en milieu scolaire doivent donc savoir ce qui se passe dans toute la famille quand ils forment l'enfant. Nous devons peut-être agir en sachant que certaines de nos pratiques peuvent rendre les frontières familiales perméables et, parfois même, les pulvériser brutalement.

Les rapports que mon équipe et moi-même avons rédigés au terme de ce projet ont finalement été compréhensifs envers les inquiétudes de la famille d'Isabel. En revanche, ce que ces rapports ont tu, c'est la tension entre les deux cultures (représentées par la famille Torres et par l'enseignante d'Isabel), ainsi que les divergences d'opinions au sein de l'équipe de recherche. Certaines questions, évidentes pour moi maintenant, n'étaient pas aussi importantes pour moi à l'époque. Il n'est pas possible de répondre aussi objectivement qu'on pourrait le croire à la question de savoir ce qui convient à un enfant donné.

\section{0 perspectives}




\section{Illustration deux : le problème des « données »; le projet des mères somaliennes}

Le cadre de cette étude de cas est celui d'une réunion de l'équipe de recherche dans une école qui vient de connaître un important afflux de familles somaliennes. Ces familles ont été acceptées dans le pays avec le statut de réfugiés politiques à cause de l'agitation qui sévissait dans leur pays d'origine. Elles vivent dans des immeubles d'une banlieue proche de l'aéroport international de Toronto.

La population de l'école s'est radicalement transformée et de nombreux enseignants ignorent totalement comment entrer en relation avec ces familles. Notre projet de recherche vise à mettre en lumière le vécu des mères des enfants qui fréquentent cette école. Comme pour tous les projets, nous devons respecter des échéances et des politiques institutionnelles, comme celles du Comité de révision déontologique, ainsi que la rédaction d'un rapport provisoire et d'un rapport final.

La personne que nous préférions pour conduire ces entrevues était un homme de race noire, originaire des Caraïbes. Sa formation est importante dans le domaine des méthodes qualitatives sur lequel il a beaucoup écrit. Nous avons compris très tôt que les hommes étaient mal à l'aise pour que cette personne conduise des entrevues avec leur femme; il a donc demandé à sa sœur de l'accompagner dans les entrevues, mais les mères n'étaient toujours pas à l'aise pour parler à un homme. Nous avons vite compris que des questions de genre étaient en jeu et que nous avions besoin de réagir.

Grâce à nos relations avec des groupes communautaires, nous avons pu embaucher Hafeezah, une musulmane, traductrice dans un centre communautaire. En plus d'un magnétophone, de formulaires de consentement éclairé et d'un protocole d'entrevue, nous avons remis à Hafeezah des articles que nous avions rédigés, lui avons exposé en détail les questions de recherche et les cadres théoriques employés. De plus, nous lui avons présenté plusieurs scénarios et jeux de rôles.

Lors de notre troisième réunion de recherche hebdomadaire, Hafeezah nous informa que tout ne se passait pas bien sur le terrain. Elle craignait même pour sa vie. Elle nous signala que de nombreuses femmes ne voulaient pas lui parler, parce qu'elle n'était pas voilée. Après en avoir longuement délibéré, nous avons décidé que notre étude serait axée sur les personnes qui avaient accepté de lui parler. Celles qui avaient invité Hafeezah chez eux voulaient passer du temps à parler des personnes de leur connaissance. Elle était attendue à des réunions de famille, devait les accompagner chez le médecin et boire beaucoup de thé.

Hafeezah a expliqué qu'étant donné ce que ces familles avaient vécu dans leur pays, où le gouvernement menace et fait souffrir les gens, elles s'inquiétaient qu'une personne associée à l'école veuille leur faire signer des papiers, prendre des notes et enregistrer leurs réponses. Il serait contre-productif de poser des questions d'entrevue tant que les familles ne pourraient pas la situer et savoir sans l'ombre d'un doute qu'elle n'était pas du mauvais bord politique.

Cela fait maintenant six semaines que nous collectons des données et nous devons rédiger un rapport d'étape pour le bailleur de fonds. Je rencontre Hafeezah pour faire avec elle le bilan des entrevues avec les familles.

Moi : Hafeezah, maintenant que vous avez eu le temps de créer de bonnes relations avec quelques familles, je souhaite savoir ce qu'elles ont dit. Commençons donc ce bilan par les bandes audio.

Hafeezah: Il n'y a pas de bandes audio. Ces mères ont vécu des situations où elles ont été trahies tant de fois. Elles se sentiraient insultées et me soupçonneraient de vouloir m'immiscer dans leur vie et d'être une indicatrice. Cette technique n'est donc pas appropriée, mais je peux vous dire ce qu'elles m'ont dit. Il vous suffit de me le demander.

Moi: Bon, parlons des formulaires de consentement signés. Nous pouvons peut-être prendre vos notes et vérifier qui vous a confié ces informations. Quelles citations pouvez-vous tirer de vos notes? Nous avons quand même besoin de véritables citations afin de pouvoir différencier ce qui s'est passé réellement de votre interprétation.

Hafeezah: Prendre des notes ou leur demander de signer d'étranges formulaires serait également un manque de respect. Je n'ai apporté ni stylo, ni papier. Nous ne signons pas de choses comme cela. Mais j'ai rédigé quelques notes en rentrant chez moi et je me souviens de ce qu'elles ont dit.

C'est à ce moment-là que j'ai compris à quel point j'avais cru connaitre, à tort, Hafeezah et sa vision du monde. J'ai pris conscience de la nécessité de disposer de directives claires pour la recherche. Le concept de responsabilité, tel que compris du monde des chercheurs, ne faisait manifestement pas partie de la vision du monde d'Hafeezah.

Je lui ai ensuite demandé de me raconter ses témoignages. Elle me dit que de nombreuses conversations avaient trait à des situations de conflit avec leurs enfants. Quand les parents essayaient d'imposer des limites et de faire régner l'ordre et le respect, les enfants menaçaient de composer le 911. Il y avait de nombreux cas d'enfants enlevés à leurs parents par les Sociétés d'aide à l'enfance, mais à cause de la confidentialité qu'elle avait promise aux familles, elle ne pouvait pas nous donner plus de détails. Hafeezah pensait que sa vie serait en danger si elle trahissait cette promesse.

\section{Interprétation de l'illustration deux}

J'étais très déçue de ne pas pouvoir accéder à ces données importantes. Cette situation soulevait des questions de qualité et d'éthique en ce qui concerne ce que nous appelons la recherche. 
La première leçon que j'ai tirée de l'expérience du chercheur canadien originaire des Caraïbes est la suivante : l'utilisation de la seule race pour servir d'identifiant ne permettrait pas de procéder à des constatations dignes d'intérêt. Les caractéristiques du groupe dont il fallait tenir compte étaient plutôt le genre, la religion, la culture et le degré d'acculturation.

La deuxième leçon que j'ai tirée avait trait à l'analyse critique de mes principes relatifs à ce que j'appelle les données ou la recherche.

Nous nous étions engagés à respecter dans notre travail les règles universitaires régissant l'éthique de la recherche réalisée avec des sujets humains. Nous devions obtenir des formulaires de consentement signés ou le consentement oral et des données suffisamment objectives pour qu'elles puissent être corroborées. ${ }^{1}$ Pourtant, en exigeant des données et en imposant une certaine méthode pour les collecter, nous avions abouti à une impasse.

Nous devions entrer dans la vie des participants, mais nous imposions des modes de pensée occidentaux en matière d'échéances et de discussions qui ont créé des obstacles à une authentique compréhension. Lutilisation du magnétophone pouvait être conceptualisée sous la forme de violence symbolique, pour employer le terme de Bourdieu. Je comprenais maintenant que les entrevues de Hafeezah ne produiraient aucune donnée utilisable aux fins de la publication d'un rapport de recherche en sciences sociales. Ce n'est pas seulement un regrettable problème de recherche. Il est réellement regrettable que la situation de ces familles et leurs opinions ne soient pas communiquées au monde extérieur, aux professionnels du terrain et aux décideurs. Bien que les protocoles de recherche de l'université aient été conçus pour protéger les familles, dans ce cas précis, ils les empêchent de participer à des recherches éducatives. Il ne s'agit pas de prôner l'abandon du rôle de protection des directives sur l'éthique de la recherche de l'université; il est impossible d'éliminer tout simplement les mesures de protection.

En étant confrontée au défi de concilier la vulnérabilité des familles et le besoin des éducateurs de comprendre la réalité vécue par les enfants et les familles, j'ai élargi mes conceptions de la recherche et du type de relations nécessaires au sein de nos équipes pour réaliser notre travail. Je trouve que la méthodologie traditionnelle est limitée et qu'elle doit être repensée de fond en comble pour obtenir des réponses authentiques.

\section{Conclusion}

Dans notre travail de recherche, nous sommes confrontés à des dilemmes difficiles à résoudre. Nous avons démontré que les problèmes de la recherche auprès des familles issues de divers horizons ne se résument pas simplement à choisir entre des méthodes qualitatives ou quantitatives, entre des approches distanciées ou participatives ou entre des chercheurs appartenant ou n'appartenant pas aux groupes étudiés. Nous avons analysé deux exemples et avons compris que les problèmes de recherche ne se résolvent pas en choisissant l'alternative « correcte» de cette dichotomie.

Comme Reid et Tom (2006) le soulignent, quand on demande aux gens de nous raconter leur vie, on leur promet implicitement d'utiliser leur témoignage de façon constructive. Pourtant, si l'équipe de recherche ne parvient pas à un accord sur les éléments essentiels qui doivent être présents pour que le compte rendu d'un témoignage constitue des « données » acceptables, nous commettons une injustice envers la famille qui nous a confié son histoire.

Cet article ne présente pas des réponses simples à des problèmes complexes. Ce que je recommande, c'est un effort de réflexion des chercheurs sur ces problèmes. Ces questions méritent de ne pas simplement être exposées dans les forums professionnels. Nous ne devrions pas nous abstenir de les traiter pour ne pas donner l'impression de maîtriser partiellement nos méthodes de recherche. Nous devons plutôt faire preuve d'humilité et de réalisme par rapport aux limites des approches actuelles.

Je laisse les lecteurs réfléchir aux aspects ou aux méthodes d'enquête qui seraient indispensables pour que nous puissions rédiger le compte rendu du vécu d'une personne ou d'une série d'événements ou de données. Ce que nous avons appris nous impose de réfléchir aux moyens à employer pour saisir le vécu de façon jugée utile et suffisamment authentique ou « objective » pour pouvoir ensuite s'en servir comme point de départ à la modification de nos pratiques.

Judith K. Bernhard est professeure à la School of Early Childhood Education de l'Université Ryerson à Toronto et directrice du programme de maîtrise en études de la petite enfance. Ses travaux de recherche ont été axés sur l'éducation de la petite enfance et les services à l'enfance et, plus particulièrement, sur l'attitude du milieu de l'éducation de la petite enfance face à l'inclusion et à la diversité. La professeure Bernhard réalise des études sur la littératie familiale transformative, les familles transnationales et le travail de collaboration entre le personnel des services à la petite enfance et les familles.

\section{Références}

BATESON, Mary Catherine. Peripheral visions: Learning along the way, New York, HarperCollins, 1994.

HOFFMAN, Eva. Lost in translation: A life in a new language, NY, Penguin Books, 1990.

REID, Colleen et Allison Tom. « Poor women's discourses of legitimacy, poverty, and health », Gender E⿱ Society, 2006, vol. 20, n³, p. 402-421.

\footnotetext{
1. Les Comités de déontologie de la recherche permettent aux chercheurs d'offrir aux participants le choix de donner leur consentement oralement ou par écrit, suivant un protocole établi. Voir à ce sujet BERNHARD, J. K. et J. E. E. YOUNG. "Gaining institutional permission: Researching precarious legal status in Canada ", Journal of Academic Ethics, 2009, vol. 7, no 3, p. 175-191.
} 

